\title{
Unraveling the spin structure of unoccupied states in $\mathrm{Bi}_{2} \mathrm{Se}_{3}$
}

\author{
Christian Datzer, ${ }^{1, *}$ Anna Zumbülte, ${ }^{1}$ Jürgen Braun, ${ }^{2}$ Tobias Förster, ${ }^{3}$ Anke B. Schmidt, ${ }^{1}$ Jianli Mi, ${ }^{4, \dagger}$ Bo Iversen, ${ }^{4}$ \\ Philip Hofmann, ${ }^{5}$ Jan Minár, ${ }^{6,2}$ Hubert Ebert, ${ }^{2}$ Peter Krüger, ${ }^{3}$ Michael Rohlfing, ${ }^{3}$ and Markus Donath ${ }^{1}$ \\ ${ }^{1}$ Physikalisches Institut, Westfälische Wilhelms-Universität Münster, Wilhelm-Klemm-Straße 10, 48149 Münster, Germany \\ ${ }^{2}$ Department of Chemistry, Ludwig-Maximilians University, Butenandtstraße 5-13, 81377 München, Germany \\ ${ }^{3}$ Institut für Festkörpertheorie, Westfälische Wilhelms-Universität Münster, Wilhelm-Klemm-Straße 10, 48149 Münster, Germany \\ ${ }^{4}$ Center for Materials Crystallography, Department of Chemistry, Interdisciplinary Nanoscience Center, \\ Aarhus University, 8000 Aarhus C, Denmark \\ ${ }^{5}$ Department of Physics and Astronomy, Interdisciplinary Nanoscience Center, Aarhus University, 8000 Aarhus C, Denmark \\ ${ }^{6}$ New Technologies Research Centre, University of West Bohemia, Univerzitni 2731, 30614 Pilzen, Czech Republic
}

(Received 17 November 2016; revised manuscript received 13 January 2017; published 1 March 2017)

\begin{abstract}
The optical control of spin currents in topological surface states opens new perspectives in (opto-) spintronics. To understand these processes, a profound knowledge about the dispersion and the spin polarization of both the occupied and the unoccupied electronic states is required. We present a joint experimental and theoretical study on the unoccupied electronic states of the topological insulator $\mathrm{Bi}_{2} \mathrm{Se}_{3}$. We discuss spin- and angle-resolved inverse-photoemission results in comparison with calculations for both the intrinsic band structure and, within the one-step model of (inverse) photoemission, the expected spectral intensities. This allows us to unravel the intrinsic spin texture of the unoccupied bands at the surface of $\mathrm{Bi}_{2} \mathrm{Se}_{3}$.
\end{abstract}

DOI: 10.1103/PhysRevB.95.115401

\section{INTRODUCTION}

In topological insulators (TIs) spin-orbit coupling (SOC) leads to a band inversion of the bulk states terminating the fundamental gap. This results in the emergence of metallic topological surface states (TSSs) crossing the gap [1,2]. These TSSs exhibit a Dirac-cone-like dispersion with a helical spin structure. $\mathrm{Bi}_{2} \mathrm{Se}_{3}(111)$ is the most prominent prototypical TI featuring a simple band structure with a single Dirac cone close to the Fermi level around the center of the surface Brillouin zone [3]. Numerous angle-resolved photoemission (ARPES) studies concentrated on this TSS with respect to its dispersion and spin texture [3-7].

Due to the interesting spin structure, TIs have emerged as promising materials in the field of spintronics and optospintronics [8-10]. Ultrafast light pulses might pave a way to control spin currents $[9,11,12]$. In this context, a profound knowledge about the electronic states above the Fermi level is crucial. Several two-photon-photoemission (2PPE) and timeand angle-resolved photoemission experiments on $\mathrm{Bi}_{2} \mathrm{Se}_{3}$ investigated the dispersion of the unoccupied states and found a second Dirac cone at $1.5 \mathrm{eV}$ [12-15]. The second Dirac cone exhibits similar characteristics as the well-known Dirac state at the Fermi level, namely, a surface characteristic, a linear dispersion, and a theoretically predicted helical spin texture.

To understand optical transitions and their dynamics, the spin texture of the electronic states below and above the Fermi level is of importance. Spin-resolved ARPES and inverse photoemission (IPE) are the tools of choice to explore these spin features. However, the interpretation of spin-polarization

*christian.datzer@uni-muenster.de

${ }^{\dagger}$ Present address: Institute for Advanced Materials, School of Material Science and Engineering, Jiangsu University, Zhenjiang 212013, China. data on materials with strong SOC is not straightforward [16-19]. The spin information measured in IPE (ARPES) may differ from the intrinsic spin polarization of the final (initial) state. It has been shown that the choice of the photon energy [11,20,21], light polarization [20-25], and experimental geometry [26] has a strong influence on the measured spin polarization of the TSS in $\mathrm{Bi}_{2} \mathrm{Se}_{3}$.

In order to determine the spin texture of the unoccupied band regime in $\mathrm{Bi}_{2} \mathrm{Se}_{3}$, we present a joint experimental and theoretical study. We show spin- and angle-resolved IPE data in comparison with calculations for both the intrinsic band structure and, within the one-step model of (inverse) photoemission, the expected IPE spectral intensities. This allows us to unravel the intrinsic spin texture of the unoccupied bands at the surface of $\mathrm{Bi}_{2} \mathrm{Se}_{3}$.

The paper is organized as follows: Our experimental approach is described in Sec. II. Several theoretical methods are introduced and compared with each other in Sec. III. Section IV is dedicated to a comparison between experimentally observed $E\left(\boldsymbol{k}_{\|}\right)$dispersion data and spectral-density calculations. In Sec. V we provide a detailed discussion of our IPE spectra in comparison with one-step-model calculations where not only energy dispersions, but also spin-dependent spectral intensities are evaluated. A summary is presented in Sec. VI.

\section{EXPERIMENTAL APPROACH}

Spin- and angle-resolved IPE is used to investigate the energy vs momentum dispersion as well as the spin structure of the electronic states above the Fermi level [27]. For excitation, an electron beam with defined energy, incidence angle $\Theta$, and spin polarization from a GaAs photocathode is directed onto the surface [Fig. 1(a)] [28]. Its spin polarization amounts to $33 \%$ and is oriented normal to the plane of incidence. The angular divergence of the electron beam leads to a momentum resolution of $\pm 0.04 \AA^{-1}$ at the Fermi energy $E_{\mathrm{F}}$ [29]. In 
(a)

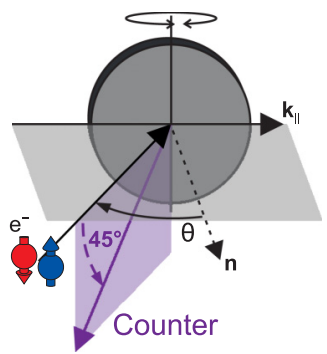

(c)
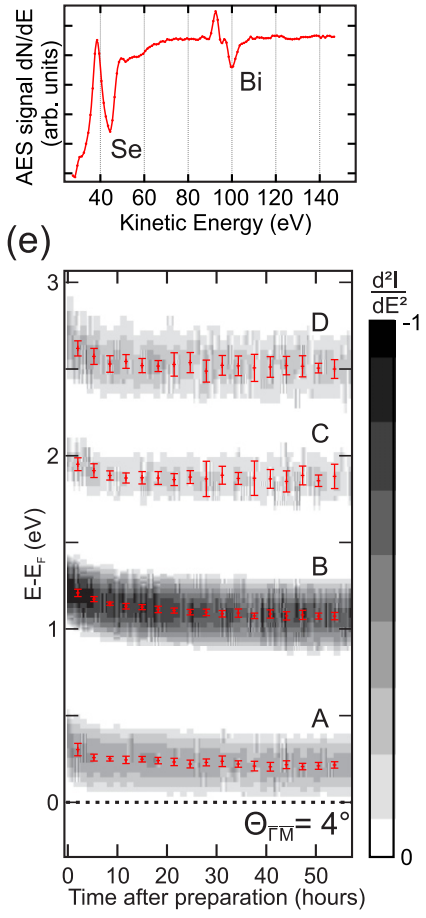

(d)
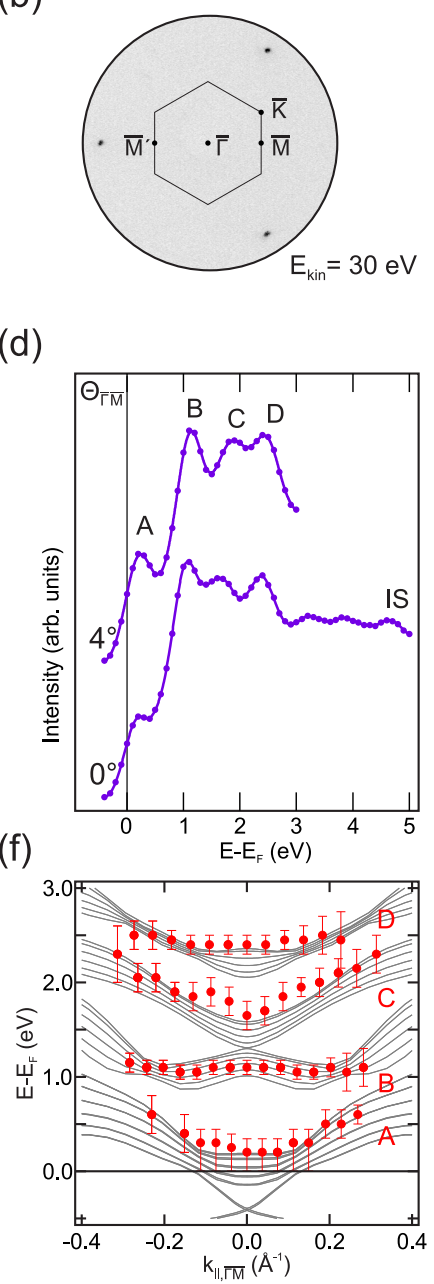

FIG. 1. (a) Experimental setup. (b) Low-energy-electrondiffraction (LEED) pattern taken at $E_{\mathrm{kin}}=30 \mathrm{eV}$, showing threefold symmetry. (c) Auger electron spectroscopy (AES) signal of an exfoliated $\mathrm{Bi}_{2} \mathrm{Se}_{3}$ sample measured with a retarding-field analyzer at a beam energy of $E_{\text {Beam }}=3 \mathrm{keV}$. (d) IPE spectra for $\Theta=0^{\circ}$ and $\Theta=4^{\circ}$ in the $\bar{\Gamma} \bar{M}$ direction. Up to an energy of $3 \mathrm{eV}$ above the Fermi edge, four structures $A-D$ are observed. For higher energies, two more features plus the image-potential state (IS) are present. (e) Time-dependent energy positions of spectral features $A-D$ : Second derivative of spectra taken at an angle of incidence of $\Theta=4^{\circ}$ where features are more clearly separated. Points mark the average peak positions of 20 spectra. (f) $E\left(\boldsymbol{k}_{\|}\right)$diagram: Experimental peak positions in the IPE spectra together with a $G W$ calculation for six quintuple layers (QLs).

the IPE process, the electrons undergo radiative transitions into lower-lying unoccupied states. The emitted photons are detected with a Geiger-Müller counter at a detection energy of $\hbar \omega=9.9 \mathrm{eV}$. The counter is located at an angle of $45^{\circ}$ below the plane of incidence in a symmetrical position for positive and negative polar angles $\Theta$ as shown in Fig. 1(a). The overall energy resolution of the experiment is $450 \mathrm{meV}$ (full width at half maximum). Spectra were taken for both spin-polarization directions of the incoming beam and are presented after normalization to a hypothetical $100 \%$ spin polarization [30]. All measurements were performed at room temperature, at a base pressure of better than $1 \times 10^{-10} \mathrm{mbar}$, and along the $\bar{M}^{\prime} \bar{\Gamma} \bar{M}$ high-symmetry direction.

High-quality single crystals of $\mathrm{Bi}_{2} \mathrm{Se}_{3}$ [31] were grown by melting stoichiometric mixtures of $5 \mathrm{~N}$-purity elemental $\mathrm{Bi}$ and $\mathrm{Se}$ at $860^{\circ} \mathrm{C}$ for $24 \mathrm{~h}$ in an evacuated quartz ampoule, cooling down to $650{ }^{\circ} \mathrm{C}$ at a rate of $2.5 \mathrm{~K} \mathrm{~h}^{-1}$, and subsequent annealing at $650^{\circ} \mathrm{C}$ for another two days. The sample then was removed to another evacuated quartz ampoule with a conical bottom, placed in a carbon crucible, and zone melted through an induction coil with a rate of $1.2 \mathrm{~mm} \mathrm{~h}^{-1}$. To prepare and clean the surface, the sample was exfoliated via the Scotch-tape method at a pressure of $5 \times 10^{-10} \mathrm{mbar}$. A LEED pattern with threefold symmetry [Fig. 1(b)] and two distinct features in AES [Fig. 1(c)] show the high quality of the sample. Caused by exfoliation, the sample surface is weakly corrugated on the millimeter scale. Since our electron beam averages over a sample area of $\approx 3 \mathrm{~mm}$ in diameter, we estimate an uncertainty of $\Theta \approx \pm 1^{\circ}$ for the IPE measurements.

Figure 1(d) displays IPE spectra of $\mathrm{Bi}_{2} \mathrm{Se}_{3}$ for normal electron incidence and for $\Theta=4^{\circ}$. In the spectra for $\Theta=$ $0^{\circ}$ four structures $(A-D)$ and an IS are dominant, whereas two further features with low intensities are visible between 3 and $4 \mathrm{eV}$. Our paper hence focuses on the energy regime between the Fermi energy and $3 \mathrm{eV}$.

ARPES experiments by Bianchi et al. [32] on the same batch of samples show intrinsic $n$ doping directly after exfoliation, leading to a Dirac point at about $250-\mathrm{meV}$ binding energy. They observed an additional time-dependent energetic shift of the features with a final binding energy of the Dirac point at about $400 \mathrm{meV}$. Several studies attribute this effect to surface reactions with various residual gases [31-42]. These reactions lead to an $n$ doping of the surface and band bending. We confirm this effect by our IPE spectra. Figure 1(e) shows the second derivative of the IPE spectra taken at an angle of incidence of $4^{\circ}$ as a function of time after exfoliation. After $20 \mathrm{~h}$, the band-bending effect is saturated, and the average value for the energetic shift of features $A-D$ amounts to about $180 \mathrm{meV}$. Our values for the time-dependent energy shift are comparable to those in the literature [32,33]. We do not observe the time-dependent development of additional quantum well states as reported in some studies [31,32,36,38]. Therefore, we assume that the entire band structure shifts rigidly and we compare our data with calculations adapted to the position of the Dirac cone as obtained from ARPES [32] after saturation. All spectra shown in this paper, including those of Fig. 1(d), have been obtained from saturated samples.

The experimentally observed peak positions of the spectral features $A-D$ for various angles of incidence have been summarized as red dots in the $E\left(\boldsymbol{k}_{\|}\right)$diagram of Fig. 1(f). Peak positions close to $E_{\mathrm{F}}$ have to be interpreted carefully. They do not necessarily reflect the real band energies because they are influenced by the temperature-dependent Fermi distribution and the finite experimental energy resolution $[30,43]$. In our case, this is relevant for structure $A$ for small angles $\Theta$ leading to large uncertainties towards $E_{\mathrm{F}}$.

The data points are compared with a $G W$ band-structure calculation for six QLs of $\mathrm{Bi}_{2} \mathrm{Se}_{3}$; details of the calculation will be given in the following section. The four dispersing spectral features resemble the calculated bands. In addition, our results are consistent with an early IPE study without angular 
resolution [44] and recent spin-integrated 2PPE measurements $[14,15]$. The main focus of our paper is the spin structure of the unoccupied bands, which will be presented in Secs. IV and $\mathrm{V}$.

\section{THEORETICAL APPROACH}

\section{A. Electronic-structure calculations}

The electronic structure of $\mathrm{Bi}_{2} \mathrm{Se}_{3}$ is well described within the $G W$ approach [45]. It starts from a local density approximation (LDA) calculation employing norm-conserving $a b$ initio pseudopotentials (PPs) in separable form [46] which include scalar-relativistic corrections and SOC [47]. Localized Gaussian orbitals are used in both LDA and $G W$ calculations, which allow for a very efficient computation of surface systems [45]. SOC is taken fully into account in the $G W$ computations. For a proper treatment of the topological surface state, a diagonalization of the $G W$ Hamiltonian turns out to be mandatory. Technical details of this methodology are given in Ref. [45].

The quasiparticle corrections distinctly influence the dispersion of the bands close to the Dirac point as can be seen from a comparison of the $G W$ and LDA derived bands in Figs. 2(a) and 2(b), respectively. In Ref. [45] we have outlined that the resulting $x$-shaped dispersion behavior of the TSS is in excellent agreement with ARPES data [48], whereas the bare LDA calculation gives a rather poor result for the part of the TSS below the Dirac point. In addition, the parabolic dispersion of the lowest conduction bands resulting from $G W$ is much closer to the experimental result in Ref. [48] than the flat density functional theory (DFT) bands. In the energy region from $0.5 \mathrm{eV}$ up to $3.0 \mathrm{eV}$, the quasiparticle corrections are of the same type as in most other systems: Conduction bands are shifted to higher energies when going from LDA to $G W$, whereas the dispersion is only slightly influenced. The resulting bands are in good agreement with structures $A-D$ seen in our IPE spectra [see Fig. 1(f)].

It turns out that the quasiparticle calculation, which is realized within the pseudopotential approach, remains difficult to handle as an input quantity in a photoemission analysis. This

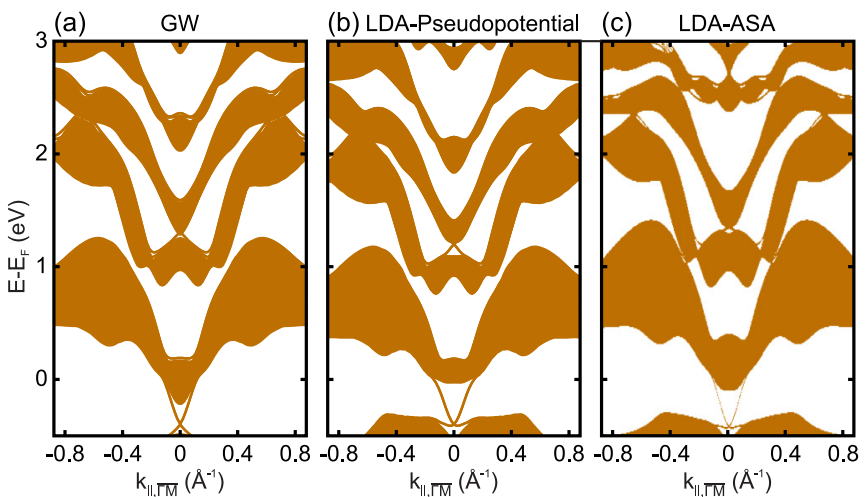

FIG. 2. Band-structure calculations for $\mathrm{Bi}_{2} \mathrm{Se}_{3}$ : (a) $G W$ calculation for six QLs, (b) LDA-DFT calculations with pseudopotentials for six QLs, and (c) atomic-sphere approximation for the half-space. All calculations have been performed for the experimental lattice parameters of Ref. [53]. analysis is used to compute IPE intensities and is typically based on Korringa-Kohn-Rostoker (KKR) multiple-scattering theory. As full-potential (FP) photoemission calculations based on space-filling relativistic KKR potentials and wave functions are not practicable for complex layered systems, such as $\mathrm{Bi}_{2} \mathrm{Se}_{3}$, we employed the atomic-sphere approximation (ASA) for the potential. The corresponding self-consistent electronic-structure calculations were performed within the $a b$ initio framework of spin-density functional theory. The Vosko et al. parametrization for the exchange and correlation potential was used [49]. The electronic structure was calculated in a fully relativistic mode by solving the corresponding Dirac equation. This was achieved using the relativistic multiple-scattering or KKR formalism in the tight-binding KKR mode [50-52]. The resulting half-space electronic structure represented by single-site scattering matrices for the different layers and the corresponding wave functions for initial- and final-state energies serve as input quantities for the corresponding calculations of the spectral intensities.

The electronic structure resulting from the relativistic multiple-scattering theory [50-52] at the LDA-ASA level for the half-space of $\mathrm{Bi}_{2} \mathrm{Se}_{3}$ is presented in Fig. 2(c). The comparison with Figs. 2(a) and 2(b) shows a good compromise between the two spectral functions based on pseudopotentials, although the theoretical approaches are quite different. This concerns, in particular, the potential construction, which is spherically symmetric in the ASA approximation and of space-filling type in the pseudopotential approach.

A significant difference between the band-structure calculations appears for higher energies around $\boldsymbol{k}_{\|}=0$ where the electronic states in the LDA-ASA calculation are higher in energy $(\approx 2.5 \mathrm{eV})$ with respect to the LDA pseudopotential results $(\approx 2.0 \mathrm{eV})$. We attribute this shift to the spherically symmetric potentials in the ASA since we obtain an analogous shift between $\mathrm{Bi}_{2} \mathrm{Se}_{3}$ bulk bands resulting from KKR calculations using the ASA and the full-potential approach. The results of the latter are in good agreement with those of the LDA pseudopotential calculation for $\mathrm{Bi}_{2} \mathrm{Se}_{3}$. As an example, we show in Table I the energy levels of the six lowest conduction bands at the $\Gamma$ point of the bulk Brillouin zone.

To get a detailed understanding of the described difference, we performed a Mulliken analysis [54] showing that the states

TABLE I. Calculated energy levels in $\mathrm{eV}$ of the lowest conduction bands of bulk $\mathrm{Bi}_{2} \mathrm{Se}_{3}$ at the $\Gamma$ point of the Brillouin zone for a rhombohedral unit cell. The LDA energies result from PP calculations and KKR calculations employing the FP approach or the ASA. For a better comparison, the energy of the lowest conduction band has been set to $0 \mathrm{eV}$ in all LDA calculations. The $G W$ results are based on quasiparticle corrections with respect to the LDA-PP results.

\begin{tabular}{lcccc}
\hline \hline Level & $G W$ & LDA-PP & LDA-FP & LDA-ASA \\
\hline 6 & 3.56 & 3.41 & 3.57 & 4.00 \\
5 & 3.09 & 2.94 & 3.03 & 3.04 \\
4 & 2.02 & 1.82 & 1.93 & 2.47 \\
3 & 1.27 & 1.21 & 1.24 & 1.47 \\
2 & 1.25 & 1.08 & 1.19 & 1.36 \\
1 & -0.23 & 0.00 & 0.00 & 0.00 \\
\hline \hline
\end{tabular}


of the LDA pseudopotential calculation at about $2.0 \mathrm{eV}$ around $\Gamma$ have distinct contributions from $\operatorname{Se} d_{x^{2}-y^{2}}$ and $\mathrm{Bi} p_{x}$ and $p_{y}$ orbitals. This result is in quantitative agreement with angular momentum-resolved density-of-states calculations which were performed within the KKR multiple-scattering theory in its full-potential version. Comparing these calculations with corresponding ones based on ASA potentials, we found that the number of $d$ states starts to increase at about $3 \mathrm{eV}$ above $E_{\mathrm{F}}$, whereas in the full-potential calculation the number of $d$ states appears to be much smaller up to energies of about $5 \mathrm{eV}$. As a consequence, the two band structures based on spherically symmetric and on space-filling cell potentials significantly deviate in their dispersion for higher energies. This shows that the impact of the non-spherical-potential components on the electronic states in complex compounds, such as $\mathrm{Bi}_{2} \mathrm{Se}_{3}$, cannot be neglected completely for higher energies.

Consequently, we infer that the KKR potential input from the LDA-ASA calculation guarantees a quantitative spectroscopical analysis in our IPE calculations for energies up to $2.5 \mathrm{eV}$ above $E_{\mathrm{F}}$, i.e., the region where the experimentally observed structures $A-C$ are energetically located. The results for higher energies, i.e., for structure $D$, should be taken with a grain of salt.

\section{B. Spectroscopical analysis}

Our spectroscopical analysis is based on the fully relativistic one-step model [55] in its spin-density-matrix formulation [56,57]. This approach allows to describe properly the complete spin-polarization vector. For the IPE calculations, in addition, we account for the surface itself by use of a Malmström-Rundgren-type surface potential [58], which can easily be included into the formalism as an additional layer. This procedure provides the correct description of the energetics and dispersion of all surface-related features. Furthermore, the relative intensities of surface states and resonances are quantitatively accounted for by calculating the corresponding matrix elements in the surface region. This procedure is described in detail, for example, in Ref. [59].

The energy-dependent retarded Green's function, which represents the final state, is obtained via the KKR. It has been calculated for a complex energy with an energy-dependent imaginary part $\operatorname{Im} V\left(E_{f}\right)=\arctan \left[0.03+0.005 / \mathrm{eV}^{2}\left(E_{f}-\right.\right.$ $\left.\left.E_{\mathrm{F}}\right)^{2}\right]$ to account for damping effects due to inelastic-scattering events. This way the finite lifetime of the final state has been considered. Also in the initial-state calculation many-body effects have been included phenomenologically by use of a parametrized weakly energy-dependent and complex inner potential $V\left(E_{i}\right)=\operatorname{Re} V\left(E_{i}\right)+i \operatorname{Im} V\left(E_{i}\right)$ as usual [60]. This generalized inner potential accounts for inelastic corrections to the elastic photocurrent [61] as well as the actual (real) inner potential, which serves as a reference energy inside the solid with respect to the vacuum level [62]. Due to the finite imaginary part $\operatorname{Im} V_{i}\left(E_{i}\right)$, the inelastic mean-free path is simulated, and thus the amplitude of the high-energy initial state can be neglected beyond a certain distance from the surface. A constant imaginary value of $\operatorname{Im} V\left(E_{i}\right)=1.0 \mathrm{eV}$ was chosen according to the measured photon energy, which lies in the vacuum-ultraviolet regime.

The spectroscopical calculations were performed for both linear $s$ - and $p$-polarized light. These intensity distributions have incoherently been superimposed according to the photon
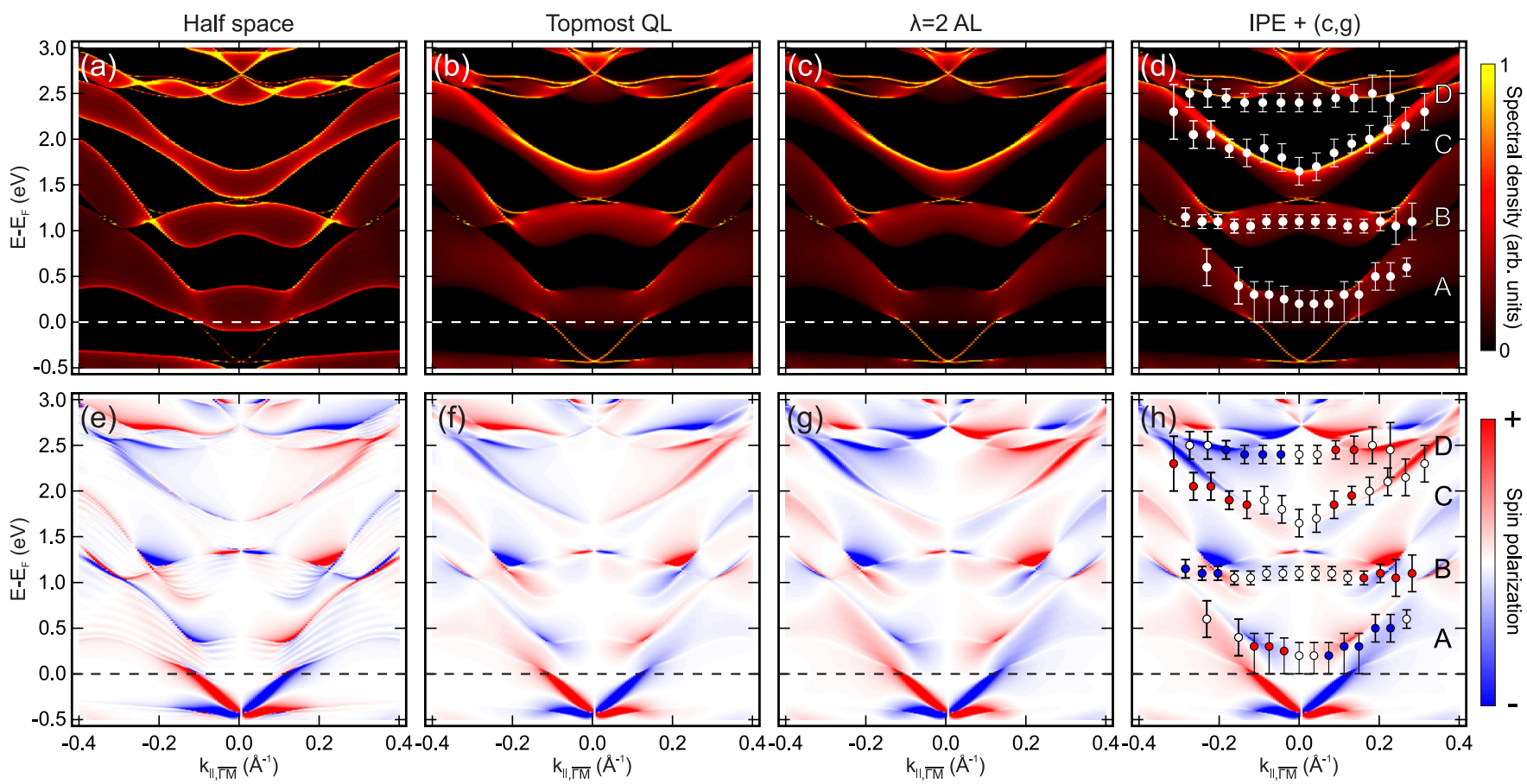

FIG. 3. Spectral densities $n\left(E, \boldsymbol{k}_{\|}\right)$of $\mathrm{Bi}_{2} \mathrm{Se}_{3}$ calculated with the LDA-ASA method (a) for the entire half-space, (b) for the topmost quintuple layer, and (c) for the surface region weighted with a probing depth of $\lambda=2$ atomic layers (ALs). (e)-(g) Corresponding spin polarization (in-plane perpendicular to $\boldsymbol{k}_{\|}$) for (a)-(c). The highest spin-polarization values $(\approx 70 \%)$ are obtained for the TSS in the calculation for the half-space in agreement with literature values $[63,64]$. (d) and (h) reproduce (c) and (d) with experimental peak positions taken from the IPE spectra added. The data points in (h) are colored where one spin channel exceeds the other. 
takeoff angle in the experiment to simulate the outgoing light, which is measured in an IPE experiment. The best agreement between experiment and theory was achieved for a photon energy of $\hbar \omega=9.2 \mathrm{eV}$, which differs by $0.7 \mathrm{eV}$ from the experimental detection energy of $9.9 \mathrm{eV}$. The reason for this difference is found in the calculated initial states, which seem to disperse at slightly lower kinetic energies, and may be explained in terms of the LDA-type ground-state electronic-structure calculation.

\section{SPECTRAL DENSITY AND $E\left(k_{\|}\right)$DATA}

We start the comparison between experiment and theory with a discussion on the calculated spectral density of $\mathrm{Bi}_{2} \mathrm{Se}_{3}$ based on the LDA-ASA method. Figure 3(a) shows the spectral density $n\left(E, \boldsymbol{k}_{\|}\right)$along $\bar{M}^{\prime} \bar{\Gamma} \bar{M}$ for the half-space. At energies higher than the TSS, four bulk bands are observed up to $3 \mathrm{eV}$. At about $1.3 \mathrm{eV}$, a second Dirac-like state connects the second and third bulk bands. Figure 3(e) shows the corresponding spin polarization. In agreement with the literature $[63,64]$, the TSS exhibits the highest polarization value of $\approx 70 \%$. Besides the TSS, considerable spin polarization is expected in the unoccupied band regime, yet with a rather complex spin texture.
The spectral-density calculations for the topmost quintuple layer [Fig. 3(b)] and for the surface region weighted with a probing depth of $\lambda=2$ ALs [Fig. 3(c)] highlight structures with surface character. Besides the TSS, surface-related spectral density (surface resonances) appears at the edges of the bulk bands, e.g., a parabolic-shaped structure at the upper edge of the third band at $\approx 1.6 \mathrm{eV}$. The calculation for the intrinsic spin structure of the topmost QL [Fig. 3(f)] resembles that of the half-space. In contrast, the spin structure changes at the very surface [Fig. 3(g)], especially around the second Dirac-like state. This effect is attributed to the existence of layer-dependent spin polarization as reported in a theoretical study for the TSS by Zhu et al. [20].

Figure 3(d) displays our experimental $E\left(\boldsymbol{k}_{\|}\right)$data in direct comparison with the spectral-density results of Fig. 3(c). Feature $A$ is attributed to the lowest unoccupied bulk band with its surface resonances plus, at least for larger $\boldsymbol{k}_{\|}$, to tails of the TSS. The TSS hybridizes with bulk bands at $\boldsymbol{k}_{\|}$ values where it appears above the Fermi level. Feature $B$ is interpreted as derived from the second bulk band around $1 \mathrm{eV}$. The calculated strong surface-related part within the third bulk band is mirrored by feature $C$. Within the energy resolution of IPE, the second Dirac state cannot be resolved. As described in (a) Experiment

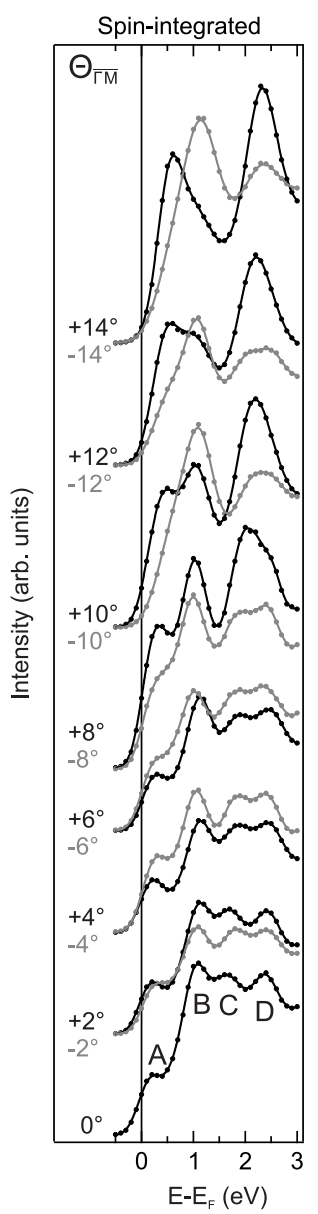

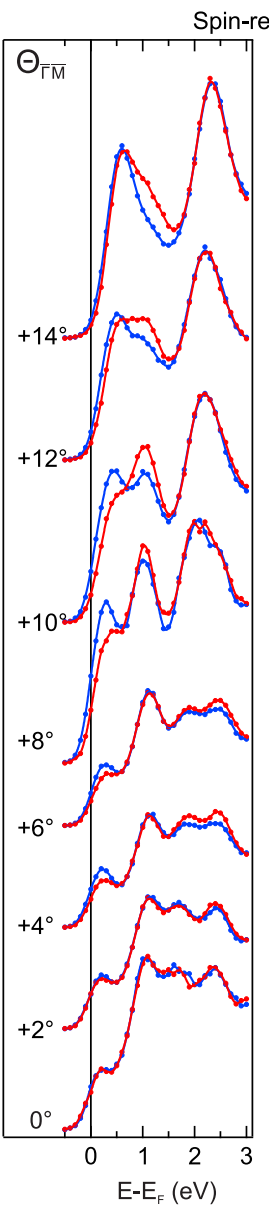

Spin-resolved

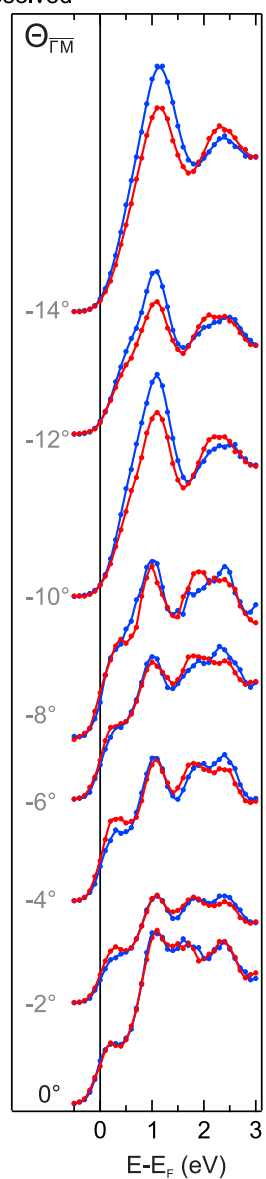

(b) Theory

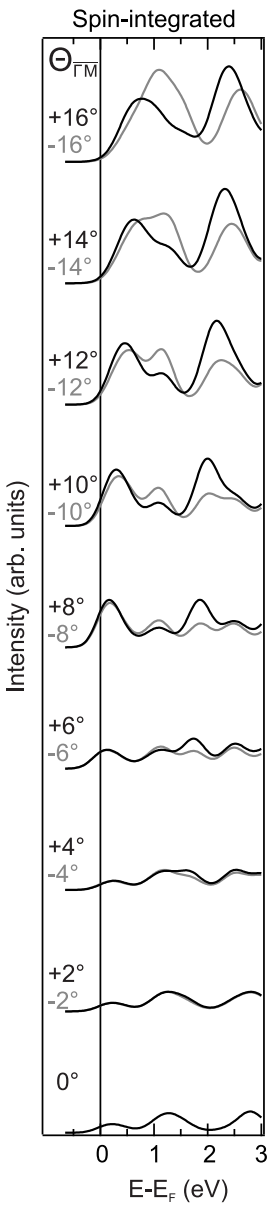

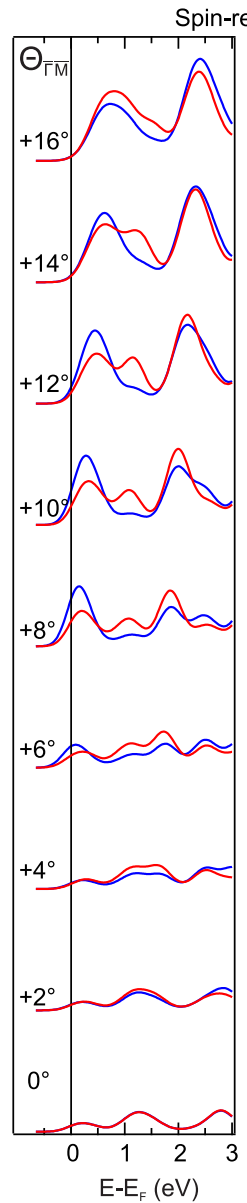

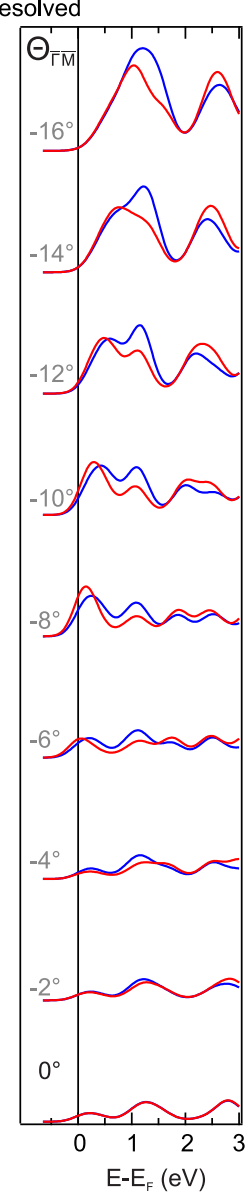

FIG. 4. (a) Spin- and angle-resolved IPE spectra along $\bar{M}^{\prime} \bar{\Gamma} \bar{M}$ (left: spin integrated; middle and right: spin resolved). (b) Corresponding calculated spectra within the one-step model for a photon energy of $\hbar \omega=9.2 \mathrm{eV}$ and a probing depth of $\lambda=2$ atomic layers. Secondary processes leading to an inelastic-background intensity are not included. 
Sec. III A, structure $D$ cannot be compared with the LDA-ASA calculations.

We now focus on the spin texture displayed in Fig. 3(h). The experimental data points are colored where one spin channel exceeds the other. The measured spin information of feature $A$ resembles the calculated spin polarization of the TSS but not of the surface resonance. We will further discuss this issue in Sec. V. Although the spin texture of $B$ fits fairly well with theory, this is clearly not the case for $C$. The spin information of $C$ does not even show the expected sign change between positive and negative $\boldsymbol{k}_{\|}$. This observation does not come as a surprise, in particular, not for bands with mixed orbital symmetries $[17,18]$. For a detailed analysis, the measured spectra have to be compared with calculations within the one-step model, which take into account the full dipole matrix elements including both initial and final states.

\section{IPE SPECTRA AND ONE-STEP-MODEL CALCULATIONS}

In this section, we extend our analysis to spin-dependent spectral intensities. We compare spin-resolved IPE spectra with one-step-model calculations as described in Sec. III B. Figure 4(a) presents our spin-integrated and spin-resolved IPE spectra for various angles of incidence $\Theta$ (from $-14^{\circ}$ to $+14^{\circ}$ ) along $\bar{M}^{\prime} \bar{\Gamma} \bar{M}$. The spectra have been accumulated from several measurements of equivalent sample preparations for the same angle of incidence. The intensities have been normalized to an equal sample current. It should be mentioned that the intensities between spectra for different angles cannot be compared directly. Different surface qualities and countercharacteristics lead to unavoidable intensity variations between the spectra. The intensity ratios between the observed features within one spectrum, however, are not affected.

The spin-integrated spectra (left panel) show the structures $A-D$. They appear with different intensity ratios for positive and negative angles. This intensity asymmetry is a consequence of the finite $k_{z}$ dependence of the structures and the missing crystal mirror symmetry between $\bar{\Gamma} \bar{M}$ and $\bar{\Gamma} \bar{M}^{\prime}$. Only surface states with a $\delta$-function-like density at the surface are expected to appear symmetrically in intensity for positive and negative angles of incidence. Obviously, the observed features $A-D$ contain contributions from bulk states or surface resonances with a finite extension into the bulk as already suggested in Sec. IV.

The theoretical spectra, spin-integrated and spin-resolved, are shown for incidence angles between $-16^{\circ}$ and $+16^{\circ}$ in Fig. 4(b). Input parameters for the calculation are a probing depth of two ALs, a Fermi function for room temperature, and an experimental energy broadening of $450 \mathrm{meV}$ but no angular divergence of the electron beam. Secondary processes leading to an inelastic background are not included in the calculations. For the experimental spectra, this background complicates the evaluation of spectral intensity ratios and the peak position determination, in particular, close to the Fermi level.

The results of our one-step-model calculations are summarized as contour plots of calculated intensities $I\left(E, \boldsymbol{k}_{\|}\right)$and spin differences $I_{\uparrow}\left(E, \boldsymbol{k}_{\|}\right)-I_{\downarrow}\left(E, \boldsymbol{k}_{\|}\right)$in Fig. 5 here without
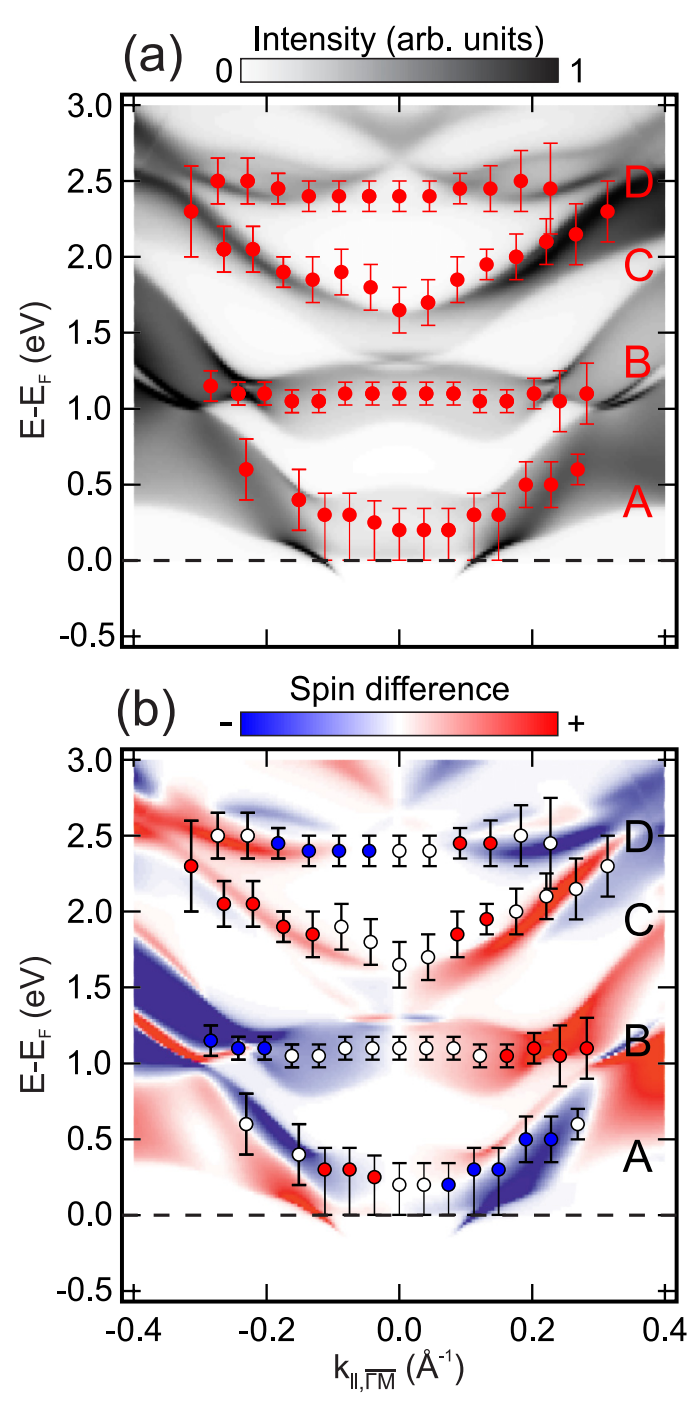

FIG. 5. Calculated IPE intensities $I\left(E, \boldsymbol{k}_{\|}\right)$of $\mathrm{Bi}_{2} \mathrm{Se}_{3}$ along $\bar{M}^{\prime} \bar{\Gamma} \bar{M}$ for (a) a photon energy of $\hbar \omega=9.2 \mathrm{eV}$ and a probing depth of $\lambda=2$ atomic layers and (b) corresponding spin differences. The experimental data points are superimposed. In (b), they are colored where one spin channel exceeds the other.

experimental broadening. The experimental data points are included for reasons of comparison.

Comparing the spin-integrated spectra in the left panels of Figs. 4(a) and 4(b) reveals an overall good agreement between experiment and theory also concerning the intensity asymmetries for positive and negative $\Theta$. However, $B$ and $C$ appear as two features in the experimental data for $\Theta=0$ and $\pm 2^{\circ}$, whereas it is one broad feature in the calculated spectra. This might be caused by the lower intensity ratio between $C$ and $B$ in the calculation compared with the experimental data.

With respect to spin-resolved data, the one-step model reproduces the experimentally observed features $A-C$ for $\Theta$ larger than $\pm 6^{\circ}$ rather well. This is true for the intensity ratios as well as spin polarizations. The discussion of feature $D$ should be neglected due to reasons mentioned in Sec. III A.

For lower $\Theta$, structure $A$ needs to be discussed in more detail. Here, $A$ is not influenced by the TSS in the calculations. Therefore, the spin texture is dominated by the bulk 
bands/surface resonances, showing the opposite sign compared with the TSS. For larger angles the spin polarization is dominated by the TSS. In contrast, our experiment detects the spin texture of the TSS even for small angles. Several reasons may account for that: (i) the limited angular resolution of the experiment, which leads to contributions of the TSS already for small angles. (ii) The dominating spectral intensity of the TSS compared with the bulk/surface resonance contributions [see Fig. 5(a)]. (iii) Any small lowering of the assumed TSS binding energy in the calculation strongly increases the TSS contribution above the Fermi level. All this leads to an enhanced influence of the TSS spin texture on structure $A$ in the IPE spectra.

For structures $B$ and $C$ good improvement is achieved by consideration of the full matrix elements in the one-step-model calculations. Especially for structure $C$ it is significant: The spectral-density calculations predict a $\boldsymbol{k}_{\|}$-dependent sign of the spin polarization [Fig. $3(\mathrm{~g})$ ], whereas our experiment and the one-step model [Fig. 5(b)] show a $\boldsymbol{k}_{\|}$-independent sign. This emphasizes the need for including both initial and final states for a proper description of the experimental results.

\section{SUMMARY}

We studied the spin-dependent electronic structure of $\mathrm{Bi}_{2} \mathrm{Se}_{3}$ above the Fermi level, both with spin- and angle- resolved inverse photoemission and with band structure calculations. From the theoretical point of view, we found out that aspherical potential components have to be included in band-structure calculations on complex materials, such as $\mathrm{Bi}_{2} \mathrm{Se}_{3}$, in order to correctly describe the band dispersion at higher energies above $E_{\mathrm{F}}$. Experimentally, the measured spin information fits in some cases the intrinsic spin polarization of the respective band, but in other cases the full excitation process has to be considered, i.e., initial state and experimental parameters that enter the matrix element. Spin- and angleresolved IPE together with state-of-the-art band-structure calculations in combination with a full description of the excitation process within the one-step model enabled us to unravel the spin texture of the unoccupied bands at the surface of $\mathrm{Bi}_{2} \mathrm{Se}_{3}$.

\section{ACKNOWLEDGMENTS}

Financial support by the BMBF Project No. 05K16WMA, the DFG SPP1666 priority program (Grants No. HO 5150/1, and No. EB154/32), and the VILLUM Centre of Excellence for Dirac Materials (Grant No. 11744) are gratefully acknowledged. J.M. acknowledges the Project VEDPMNF (Project No CZ.02.1.01/0.0/0.0/15.003/0000358) of the Czech ministerium MSMT. The work has also benefited from discussions within the COST Action (Grant No. MP 1306 EUSpec).
[1] M. Z. Hasan and C. L. Kane, Rev. Mod. Phys. 82, 3045 (2010).

[2] X.-L. Qi and S.-C. Zhang, Rev. Mod. Phys. 83, 1057 (2011).

[3] Y. Xia, D. Qian, D. Hsieh, L. Wray, A. Pal, H. Lin, A. Bansil, D. Grauer, Y. S. Hor, R. J. Cava, and M. Z. Hasan, Nat. Phys. 5, 398 (2009).

[4] H. Zhang, C.-X. Liu, X.-L. Qi, X. Dai, Z. Fang, and S.-C. Zhang, Nat. Phys. 5, 438 (2009).

[5] D. Hsieh, Y. Xia, D. Qian, L. Wray, J. H. Dil, F. Meier, J. Osterwalder, L. Patthey, J. G. Checkelsky, N. P. Ong, A. V. Fedorov, H. Lin, A. Bansil, D. Grauer, Y. S. Hor, R. J. Cava, and M. Z. Hasan, Nature (London) 460, 1101 (2009).

[6] C. Cacho, A. Crepaldi, M. Battiato, J. Braun, F. Cilento, M. Zacchigna, M. C. Richter, O. Heckmann, E. Springate, Y. Liu, S. S. Dhesi, H. Berger, P. Bugnon, K. Held, M. Grioni, H. Ebert, K. Hricovini, J. Minár, and F. Parmigiani, Phys. Rev. Lett. 114, 097401 (2015).

[7] C. Jozwiak, J. A. Sobota, K. Gotlieb, A. F. Kemper, C. R. Rotundu, R. J. Birgeneau, Z. Hussain, D.-H. Lee, Z.-X. Shen, and A. Lanzara, Nat. Commun. 7, 13143 (2016).

[8] P. Hosur, Phys. Rev. B 83, 035309 (2011).

[9] J. W. McIver, D. Hsieh, H. Steinberg, P. Jarillo-Herrero, and N. Gedik, Nat. Nanotechnol. 7, 96 (2012).

[10] D. Pesin and A. H. MacDonald, Nat. Mater. 11, 409 (2012).

[11] J. Sánchez-Barriga, A. Varykhalov, J. Braun, S.-Y. Xu, N. Alidoust, O. Kornilov, J. Minár, K. Hummer, G. Springholz, G. Bauer, R. Schumann, L. V. Yashina, H. Ebert, M. Z. Hasan, and O. Rader, Phys. Rev. X 4, 011046 (2014).

[12] M. C. Wang, S. Qiao, Z. Jiang, S. N. Luo, and J. Qi, Phys. Rev. Lett. 116, 036601 (2016).
[13] D. Niesner, T. Fauster, S. V. Eremeev, T. V. Menshchikova, Y. M. Koroteev, A. P. Protogenov, E. V. Chulkov, O. E. Tereshchenko, K. A. Kokh, O. Alekperov, A. Nadjafov, and N. Mamedov, Phys. Rev. B 86, 205403 (2012).

[14] J. A. Sobota, S.-L. Yang, A. F. Kemper, J. J. Lee, F. T. Schmitt, W. Li, R. G. Moore, J. G. Analytis, I. R. Fisher, P. S. Kirchmann, T. P. Devereaux, and Z.-X. Shen, Phys. Rev. Lett. 111, 136802 (2013).

[15] D. Niesner, S. Otto, T. Fauster, E. V. Chulkov, S. V. Eremeev, O. E. Tereshchenko, and K. A. Kokh, J. Electron Spectrosc. Relat. Phenom. 195, 258 (2014).

[16] J. Henk, A. Ernst, and P. Bruno, Phys. Rev. B 68, 165416 (2003).

[17] S. N. P. Wissing, A. B. Schmidt, H. Mirhosseini, J. Henk, C. R. Ast, and M. Donath, Phys. Rev. Lett. 113, 116402 (2014).

[18] H. Wortelen, H. Mirhosseini, K. Miyamoto, A. B. Schmidt, J. Henk, and M. Donath, Phys. Rev. B 91, 115420 (2015).

[19] K. Miyamoto, H. Wortelen, H. Mirhosseini, T. Okuda, A. Kimura, H. Iwasawa, K. Shimada, J. Henk, and M. Donath, Phys. Rev. B 93, 161403(R) (2016).

[20] Z.-H. Zhu, C. N. Veenstra, G. Levy, A. Ubaldini, P. Syers, N. P. Butch, J. Paglione, M. W. Haverkort, I. S. Elfimov, and A. Damascelli, Phys. Rev. Lett. 110, 216401 (2013).

[21] Z.-H. Zhu, C. N. Veenstra, S. Zhdanovich, M. P. Schneider, T. Okuda, K. Miyamoto, S.-Y. Zhu, H. Namatame, M. Taniguchi, M. W. Haverkort, I. S. Elfimov, and A. Damascelli, Phys. Rev. Lett. 112, 076802 (2014).

[22] C. Jozwiak, Y. L. Chen, A. V. Fedorov, J. G. Analytis, C. R. Rotundu, A. K. Schmid, J. D. Denlinger, Y.-D. Chuang, D.-H. Lee, I. R. Fisher, R. J. Birgeneau, Z.-X. Shen, Z. Hussain, and A. Lanzara, Phys. Rev. B 84, 165113 (2011). 
[23] C.-H. Park and S. G. Louie, Phys. Rev. Lett. 109, 097601 (2012).

[24] C. Jozwiak, C.-H. Park, K. Gotlieb, C. Hwang, D.-H. Lee, S. G. Louie, J. D. Denlinger, C. R. Rotundu, R. J. Birgeneau, Z. Hussain, and A. Lanzara, Nat. Phys. 9, 293 (2013).

[25] K. Kuroda, K. Yaji, M. Nakayama, A. Harasawa, Y. Ishida, S. Watanabe, C.-T. Chen, T. Kondo, F. Komori, and S. Shin, Phys. Rev. B 94, 165162 (2016).

[26] C. Seibel, J. Braun, H. Maßß, H. Bentmann, J. Minár, T. V. Kuznetsova, K. A. Kokh, O. E. Tereshchenko, T. Okuda, H. Ebert, and F. Reinert, Phys. Rev. B 93, 245150 (2016).

[27] M. Donath, J. Phys.: Condens. Matter 11, 9421 (1999).

[28] U. Kolac, M. Donath, K. Ertl, H. Liebl, and V. Dose, Rev. Sci. Instrum. 59, 1933 (1988)

[29] A. Zumbülte, A. B. Schmidt, and M. Donath, Rev. Sci. Instrum. 86, 013908 (2015); 86, 069901 (2015).

[30] M. Donath, Appl. Phys. A 49, 351 (1989).

[31] M. Bianchi, D. Guan, S. Bao, J. Mi, B. B. Iversen, P. D. King, and P. Hofmann, Nat. Commun. 1, 128 (2010).

[32] M. Bianchi, R. C. Hatch, D. Guan, T. Planke, J. Mi, B. B. Iversen, and P. Hofmann, Semicond. Sci. Technol. 27, 124001 (2012).

[33] H. M. Benia, C. Lin, K. Kern, and C. R. Ast, Phys. Rev. Lett. 107, 177602 (2011).

[34] M. S. Bahramy, P. D. C. King, A. de la Torre, J. Chang, M. Shi, L. Patthey, G. Balakrishnan, P. Hofmann, R. Arita, N. Nagaosa, and F. Baumberger, Nat. Commun. 3, 1159 (2012).

[35] C. Chen, S. He, H. Weng, W. Zhang, L. Zhao, H. Liu, X. Jia, D. Mou, S. Liu, J. He, Y. Peng, Y. Feng, Z. Xie, G. Liu, X. Dong, J. Zhang, X. Wang, Q. Peng, Z. Wang, S. Zhang, F. Yang, C. Chen, Z. Xu, X. Dai, Z. Fang, and X. J. Zhou, Proc. Natl. Acad. Sci. U.S.A. 109, 3694 (2012).

[36] T. Förster, P. Krüger, and M. Rohlfing, Phys. Rev. B 91, 035313 (2015).

[37] X. He, W. Zhou, Z. Y. Wang, Y. N. Zhang, J. Shi, R. Q. Wu, and J. A. Yarmoff, Phys. Rev. Lett. 110, 156101 (2013).

[38] P. D. C. King, R. C. Hatch, M. Bianchi, R. Ovsyannikov, C. Lupulescu, G. Landolt, B. Slomski, J. H. Dil, D. Guan, J. L. Mi, E. D. L. Rienks, J. Fink, A. Lindblad, S. Svensson, S. Bao, G. Balakrishnan, B. B. Iversen, J. Osterwalder, W. Eberhardt, F. Baumberger, and P. Hofmann, Phys. Rev. Lett. 107, 096802 (2011).

[39] K. Park, C. D. Beule, and B. Partoens, New J. Phys. 15, 113031 (2013).

[40] S. Roy, H. L. Meyerheim, A. Ernst, K. Mohseni, C. Tusche, M. G. Vergniory, T. V. Menshchikova, M. M. Otrokov, A. G. Ryabishchenkova, Z. S. Aliev, M. B. Babanly, K. A. Kokh, O. E. Tereshchenko, E. V. Chulkov, J. Schneider, and J. Kirschner, Phys. Rev. Lett. 113, 116802 (2014).

[41] T. Valla, Z.-H. Pan, D. Gardner, Y. S. Lee, and S. Chu, Phys. Rev. Lett. 108, 117601 (2012).
[42] D. D. dos Reis, L. Barreto, M. Bianchi, G. A. S. Ribeiro, E. A. Soares, W. S. e. Silva, V. E. de Carvalho, J. Rawle, M. Hoesch, C. Nicklin, W. P. Fernandes, J. Mi, B. B. Iversen, and P. Hofmann, Phys. Rev. B 88, 041404(R) (2013).

[43] S. D. Stolwijk, A. B. Schmidt, and M. Donath, Phys. Rev. B 82, 201412 (2010).

[44] Y. Ueda, A. Furuta, H. Okuda, M. Nakatake, H. Sato, H. Namatame, and M. Taniguchi, J. Electron Spectrosc. Relat. Phenom. 101, 677 (1999).

[45] T. Förster, P. Krüger, and M. Rohlfing, Phys. Rev. B 92, 201404 (2015).

[46] L. Kleinman and D. M. Bylander, Phys. Rev. Lett. 48, 1425 (1982).

[47] B. Stärk, P. Krüger, and J. Pollmann, Phys. Rev. B 84, 195316 (2011).

[48] G. Landolt, S. Schreyeck, S. V. Eremeev, B. Slomski, S. Muff, J. Osterwalder, E. V. Chulkov, C. Gould, G. Karczewski, K. Brunner, H. Buhmann, L. W. Molenkamp, and J. H. Dil, Phys. Rev. Lett. 112, 057601 (2014).

[49] S. H. Vosko, L. Wilk, and M. Nusair, Can. J. Phys. 58, 1200 (1980).

[50] H. Ebert, in Electronic Structure and Physical Properties of Solids, edited by H. Dreyssé (Springer, Berlin, 2000), Vol. 535, p. 191.

[51] H. Ebert, D. Ködderitzsch, and J. Minár, Rep. Prog. Phys. 74, 096501 (2011).

[52] H. Ebert, "The Munich SPR-KKR package" (2012).

[53] C. Pérez Vicente, J. L. Tirado, K. Adouby, J. C. Jumas, A. A. Touré, and G. Kra, Inorg. Chem. 38, 2131 (1999).

[54] R. S. Mulliken, J. Chem. Phys. 23, 1833 (1955).

[55] J. Braun, Rep. Prog. Phys. 59, 1267 (1996).

[56] S. V. Halilov, E. Tamura, D. Meinert, H. Gollisch, and R. Feder, J. Phys.: Condens. Matter 5, 3859 (1993).

[57] J. Braun, K. Miyamoto, A. Kimura, T. Okuda, M. Donath, H. Ebert, and J. Minár, New J. Phys. 16, 015005 (2014).

[58] G. Malmström and J. Rundgren, Comput. Phys. Commun. 19, 263 (1980).

[59] A. Nuber, J. Braun, F. Forster, J. Minár, F. Reinert, and H. Ebert, Phys. Rev. B 83, 165401 (2011).

[60] J. B. Pendry, Low Energy Electron Diffraction (Academic, London, 1974).

[61] G. Borstel, Appl. Phys. A 38, 193 (1985).

[62] G. Hilgers, M. Potthoff, N. Müller, U. Heinzmann, L. Haunert, J. Braun, and G. Borstel, Phys. Rev. B 52, 14859 (1995).

[63] O. V. Yazyev, J. E. Moore, and S. G. Louie, Phys. Rev. Lett. 105, 266806 (2010).

[64] Y. Zhao, Y. Hu, L. Liu, Y. Zhu, and H. Guo, Nano Lett. 11, 2088 (2011). 